\title{
SENSITIVITY ANALYSIS OF TIME/DEPTH DEPENDENT CHLORIDE DIFFUSION COEFFICIENT IN CONCRETE
}

\author{
Yung-Ming Sun \\ Department of Construction Technology, Tungnan University, Taipei, Taiwan, R.O.C., ymsun@mail.tnu.edu.tw \\ Ta-Peng Chang \\ Department of Construction Engineering, Green Building Materials Center, National Taiwan University of Science and \\ Technology, Taipei, Taiwan, R.O.C. \\ Ming-Te Liang \\ Department of Civil Engineering, China University of Science and Technology, Taipei, Taiwan, R.O.C.
}

Follow this and additional works at: https://jmstt.ntou.edu.tw/journal

Part of the Engineering Commons

\section{Recommended Citation}

Sun, Yung-Ming; Chang, Ta-Peng; and Liang, Ming-Te (2011) "SENSITIVITY ANALYSIS OF TIME/DEPTH DEPENDENT CHLORIDE DIFFUSION COEFFICIENT IN CONCRETE," Journal of Marine Science and Technology: Vol. 19: Iss. 6, Article 10.

DOI: $10.51400 / 2709-6998.2208$

Available at: https://jmstt.ntou.edu.tw/journal/vol19/iss6/10

This Research Article is brought to you for free and open access by Journal of Marine Science and Technology. It has been accepted for inclusion in Journal of Marine Science and Technology by an authorized editor of Journal of Marine Science and Technology. 


\title{
SENSITIVITY ANALYSIS OF TIME/DEPTH DEPENDENT CHLORIDE DIFFUSION COEFFICIENT IN CONCRETE
}

\author{
Yung-Ming Sun*, Ta-Peng Chang**, and Ming-Te Liang***
}

Key words: chloride diffusion, sensitivity analysis, concrete, nonsteady state.

\begin{abstract}
This paper presents the results for a sensitivity analysis of the time/depth dependent chloride diffusion model in concrete using differential analysis techniques. Four governing prameters of the model are taken to determine the sensitivity of the chloride diffusion coefficient: the time of exposure, inspecting depth, surface chloride concentration, and chloride concetration at the inspecting point. The results of this sensitivity analysis are discussed in detail, including variations in the diffusion coefficient according to the four parameters and the implications for durability design of concrete structures.
\end{abstract}

\section{INTRODUCTION}

Durability analyses of RC structures built in chloride environments are mostly based on assuming Fick's law of diffusion [10] when predicting the time and space variations of the chloride content in concrete and using the concept of a chloride threshold to define the corrosion resistance of reinforcing steel to chloride attack [4]. The chloride diffusion coefficient is an indicator of the accessibility of concrete to chloride penetration and can be used for durability design of $\mathrm{RC}$ structures. The governing parameters for calculating the chloride diffusion coefficient include the time of exposure to a chloride environment, inspecting depth, surface chloride concentration, and chloride concentration at the inspecting point. In practical applications, the durability design of RC structures usually specifies a minimum concrete depth depending on the

Paper submitted 03/01/10; revised 06/03/10; accepted 06/11/10. Author for correspondence: Yung-Ming Sun (e-mail: ymsun@mail.tnu.edu.tw).

*Department of Construction Technology, Tungnan University, Taipei, Taiwan, R.O.C.

**Department of Construction Engineering, Green Building Materials Center, National Taiwan University of Science and Technology, Taipei, Taiwan, R.O.C.

***Department of Civil Engineering, China University of Science and Technology, Taipei, Taiwan, R.O.C. seriousness of chloride corrosion damage, a maximum water to cement (w/c) ratio to achieve low diffusivity, and the use of corrosion-resistant reinforcing steels.

However, the above four parameters have considerable levels of uncertainty. This uncertainty in the diffusion model may cause over- or underestimations of the service life, insufficient planning for inspection and maintenance, and increased engineering costs. Alonso et al. [1] suggested the following sources of uncertainty: (i) concrete mixing and aging causes time and space variations in the chloride diffusivity; (ii) quality control for the engineering, workmanship, and size of the structure cause uncertainty in the concrete cover depth; (iii) the severity of the chloride environment causes variations in the surface chloride concentration; and (iv) the types of reinforcing steel, concrete mixture, and test method used cause uncertainty in the chloride threshold concentration for initiating corrosion of the reinforcing steel. Consequently, the combination of these variables leads to considerable uncertainty in diffusion model results. Therefore, a sensitivity analysis is necessary to estimate the impact of uncertainties in the input parameters on the uncertainty of the diffusion model. Several sensitivity studies in the literature dealt with the performance of concrete structures and evaluated the impact of input parameter uncertainties on the output results of the diffusion model [2, 7-9, 11]. Oh and Yang [5] used the Latin hypercube simulation technique to predict the variability for the longterm response of a structure. Hohenbichler and Rackwitz [3] defined the parametric sensitivity factor for the first-order reliability index $\beta$ with respect to the problem parameter $\theta$ as the derivative $d \beta / d \theta$.

In this study, a sensitivity analysis of the time/depth dependent diffusion model using the differential analysis technique was performed to identify the most significant parameters and quantify their impacts on the chloride diffusion coefficient [4]. Variations in the chloride diffusion coefficient were evaluated by how it was affected by variations in the input data of the model, which include the time of exposure to chloride environment, inspected depth, surface chloride concentration, and chloride concentration at the inspecting point. Sensitivity analysis results can provide valuable insights and better understanding of the chloride diffusivity of concrete and its governing parameters. Furthermore, sensitivity analysis 
can be used to identify the importance of uncertainties in the diffusion model input when prioritizing additional data collection or research on parameters found to be significant.

\section{TIME/DEPTH DEPENDENT CHLORIDE DIFFUSION MODEL}

For non-steady state of diffusion, chloride diffuses into the concrete that changes the chloride concentration $C$ at different time $t$ and different location $x$ of the concrete. The analytical solution of Fick's second law of non-steady state of diffusion was derived by Sun et al. [6] as follows:

$$
\frac{\int_{C_{i}}^{C_{r}} D(C(x, t)) d C}{\int_{C_{i}}^{C_{s}} D(C(x, t)) d C}=\operatorname{erf} c \frac{x}{\sqrt{4 D t}}
$$

where $x$ is the penetration depth from the structure surface, and $t$ is the exposure time, $C(x, t)$ is the total chloride concentration, $D(C(x, t))$ is the apparent diffusion coefficient dependent on chloride concentration $C(x, t), C_{i}$ is the initial chloride concentration of the concrete, $C_{s}$ is the surface concentration. For the purpose of brevity, the state variables in $C(x, t)$ and $D(C(x, t))$ will be omitted hereinafter.

And, the complementary error function is denoted by:

$$
\operatorname{erfc} \frac{x}{\sqrt{4 D t}}=1-\frac{2}{\sqrt{\pi}} \int_{0}^{\frac{x}{\sqrt{4 D t}}} e^{-\eta^{2}} d \eta
$$

Transforming Crank's solution for the chloride concentration in concrete into a deterministic model of the diffusion coefficient produces an explicit function with the parameters $t$, $d_{c}, C_{s}$, and $C_{r}$ as follows:

$$
D=f\left(t, d_{c}, C_{s}, C_{r}\right)=\frac{d_{c}^{2}}{4 t\left[\operatorname{erfc}^{-1}\left(\frac{\int_{C_{i}}^{C_{r}} D(C) d C}{\int_{C_{i}}^{C_{s}} D(C) d C}\right)\right]^{2}}
$$

where $d_{c}$ is the inspecting depth of concrete.

These governing parameters can be categorizes as follows: (i) the time parameter $t$ denotes the exposure, maturity, and aging periods of concrete and is also used to estimate the service life of concrete; (ii) the structural parameter $d_{c}$ is the inspecting depth, where the service life of a structure is assessed by inspection at the concrete cover depth; (iii) the environmental parameter $C_{s}$ denotes the severity of chloride encroaching and the chloride content of the surrounding environment; and (iv) the material parameter $C_{r}$ denotes the quantity of chloride that penetrated into concrete at a certain time and depth.

\section{DIFFERENTIAL SENSITIVITY ANALYSIS}

In this study, the differential analysis method [4] was used to investigate the sensitivity of the diffusion coefficient in the time/depth dependent diffusion model to the four basic parameters of concrete structures exposed to chloride. This method is based on using a Taylor series to approximate the model under consideration. The time/depth dependent diffusion model shown in Eq. (3) can be represented as the following function:

$$
D=f\left(t, d_{c}, C_{s}, C_{r}\right)=f\left(X_{1}, X_{2}, X_{3}, X_{4}\right)
$$

where the governing parameters are the input variables and are represented by the vector:

$$
X=\left[X_{1}, X_{2}, X_{3}, X_{4}\right]
$$

A first-order Taylor series approximation of $D$ has the following form, with $X_{0}$ representing a base vector:

$$
D(X) \cong D\left(X_{0}\right)+\sum_{j=1}^{4} \frac{\partial f\left(X_{0}\right)}{\partial X_{j}}\left(X_{j}-X_{j 0}\right)
$$

where $X_{j}$ is the $j$ th parameter of the model and $X_{j 0}$ is the base (or mean) value of the $j$ th parameter of the model.

The values of the partial derivatives measure the local sensitivity. The order of approximation depends on the curvature of the surface $D=f(X)$. Eq. (3) can be rewritten in the following form:

$$
\frac{D(X)-D\left(X_{0}\right)}{D\left(X_{0}\right)}=\sum_{j=1}^{4} \frac{\partial f\left(X_{0}\right)}{\partial X_{j}} \frac{X_{j 0}}{D_{i}\left(X_{0}\right)} \frac{\left(X_{j}-X_{j 0}\right)}{X_{j 0}}
$$

Let

$$
\begin{gathered}
\Delta D=D(X)-D\left(X_{0}\right) \\
\Delta X_{j}=X_{j}-X_{j 0}
\end{gathered}
$$

and

$$
D\left(X_{0}\right)=D_{0}
$$

In this approach, the variability of $D$ or $f(X)$ can be inferred by changing one parameter of one factor at a time and keeping the other parameters constant while noting the change in $D$; therefore,

$$
\frac{\partial f}{\partial x_{k}}=0 \quad k=1,2, \ldots, n \text { where } k \neq j
$$


and

$$
\frac{\partial f}{\partial x_{j}}=f^{\prime}\left(x_{j}\right)
$$

Therefore, Eq. (7) becomes:

$$
\frac{\Delta D}{\Delta X_{j}} \frac{X_{j 0}}{D_{0}}=\frac{\partial f\left(X_{0}\right)}{\partial X_{j}} \frac{X_{j 0}}{D_{0}}=S\left(X_{j}\right)=S_{j}
$$

where $S_{j}$ is the normalized first-order sensitivity coefficient of $D$ to $X_{j}$, which provides a measure of the relative change in $D$ that results from a relative change in $X_{j}$ when the other variables are kept constant. The change or perturbation in $X_{j}$ should be small.

$$
S_{j}=\frac{\partial f\left(X_{0}\right)}{\partial X_{j}} \frac{X_{j 0}}{D_{0}} \quad j=1, \ldots, 4
$$

Higher order expressions can also be derived. Given the assumption of one variable parameter at a time, it is possible to include higher order terms of the Taylor series approximation to determine the higher order sensitivity coefficients $\operatorname{Sh}\left(X_{j}\right)$ through the following equation:

$$
\begin{aligned}
\Delta D & =f\left(X_{j}+\Delta X_{j}\right)-f\left(X_{j}\right) \\
& =f^{\prime}\left(X_{j}\right) \Delta X_{j}+\ldots+\frac{f^{n}\left(X_{j}\right)}{n !}\left(\Delta X_{j}\right)^{n}+\frac{f^{n+1}\left(\theta X_{j}\right)}{(n+1) !}\left(\Delta X_{j}\right)^{n+1}
\end{aligned}
$$

where fn denotes the nth order derivative of $f\left(X_{j}\right)$ for $X_{j}<$ $\theta X_{j}<X_{j}+\Delta X_{j}$. Substituting Eq. (11) into Eq. (10b) yields:

$$
\begin{aligned}
S_{h j} & =S_{h}\left(X_{j}\right) \\
& =\frac{X_{j 0}}{D_{0}}\left[f^{\prime}\left(X_{j 0}\right)+\ldots+\frac{f^{n}\left(X_{j 0}\right)}{n !}\left(\Delta X_{j}\right)^{n}+\frac{f^{n+1}\left(\theta X_{j}\right)}{(n+1) !}\left(\Delta X_{j}\right)^{n}\right]
\end{aligned}
$$

Using Eq. (10b), the first-order sensitivity coefficients of $D$ to $t, d_{c}, C_{s}, C_{r}$, can be obtained as follows:

$$
\begin{gathered}
S(t)=f^{\prime}(t) \frac{t}{D}=-1 \\
S\left(d_{c}\right)=f^{\prime}\left(d_{c}\right) \frac{d_{c}}{D}=2
\end{gathered}
$$

Let

$$
D(C)=A \times e^{-B \times C}
$$

$$
g\left(C_{s}, C_{r}\right)=\operatorname{erfc}^{-1}\left(\frac{\int_{C_{i}}^{C_{r}} D(C) d C}{\int_{C_{i}}^{C_{s}} D(C) d C}\right)
$$

Then

$$
\begin{gathered}
S\left(C_{s}\right)=f^{\prime}\left(C_{s}\right) \frac{C_{s}}{D}=\frac{C_{s} A e^{-B C_{s}+g^{2}} \int_{C_{i}}^{C_{r}} D(C) d C \sqrt{\pi}}{g\left(\int_{C_{i}}^{C_{s}} D(C) d C\right)^{2}} \\
S\left(C_{r}\right)=f^{\prime}\left(C_{r}\right) \frac{C_{r}}{D}=\frac{C_{r} A e^{-B C_{r}+g^{2}} \sqrt{\pi}}{g \int_{C_{i}}^{C_{s}} D(C) d C}
\end{gathered}
$$

The first-order sensitivity coefficients of $D$ to $t$ and $d_{c}$ were easily computed and found to be constant. However, the first-order sensitivity coefficients of $D$ to $C_{s}$ or $C_{r}$ are complex functions of the variables $C_{s}$ and $C_{r}$, so they are higher order sensitivity coefficients.

The higher order derivatives of $D$ to $t$ or $d_{c}$ were easily obtained; thus, high-order sensitivity coefficients $\operatorname{Sh}(D)$ and $\operatorname{Sh}\left(d_{c}\right)$ of $D$ to $t$ and $d_{c}$ can be obtained as follows:

$$
\begin{gathered}
\frac{\Delta D}{D}=-\frac{\frac{\Delta t}{t}}{\frac{\Delta t}{t}+1} \\
S_{h}(t)=-\frac{1}{\frac{\Delta t}{t}+1} \\
\frac{\Delta D}{D}=2 \frac{\Delta d_{c}}{d_{c}}+\left(\frac{\Delta d_{c}}{d_{c}}\right)^{2} \\
S_{h}\left(d_{c}\right)=2+\frac{\Delta d_{c}}{d_{c}}
\end{gathered}
$$

The chloride diffusion coefficient is a material property of concrete that is primarily dependent on the pore structure and saturation of the pore solution. The four variables $t, d_{c}, C_{s}$, and $C_{r}$ may be correlated because all of them are related to concrete properties. However, the four variables were assumed to be independent variables in this study to illustrate the dependence of $D$ on each of them.

\section{RESULTS OF SENSITIVITY ANALYSIS}

\section{Sensitivity of Chloride Diffusion Coefficient $D$ to Time of Exposure $t$}

Eqs. (19) and (20) are used to evaluate the effect of a rela- 


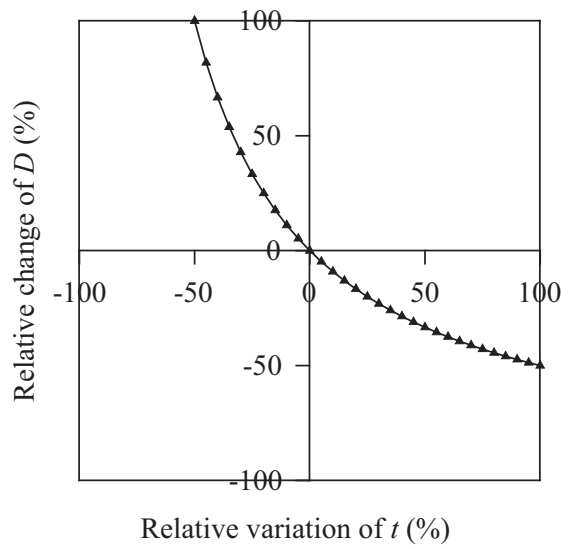

Fig. 1. Higher order impact of $\Delta D / D$ against $\Delta t / t$.

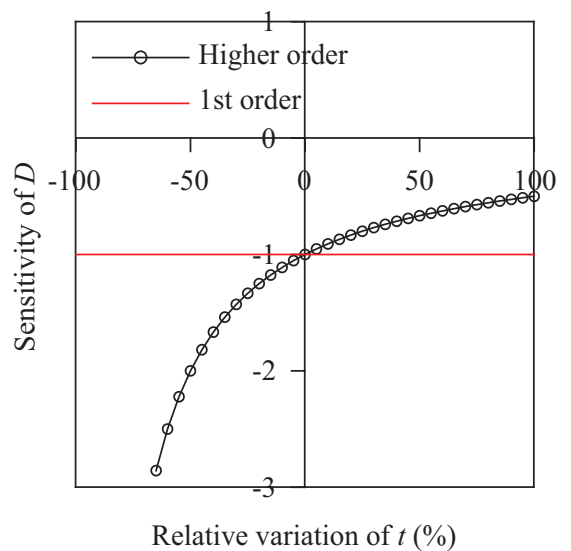

Fig. 2. Sensitivity of chloride diffusion coefficient $D$ to time $t$.

tive change in $\mathrm{t}$ (denoted by $\Delta t / t$ ) on $D$, the impact of $\Delta D / D$ to $\Delta t / t$ is plotted in Fig. 1, and the sensitivity of the coefficient $D$ to time $t$ is shown in Fig. 2. The resulting two main observations are as follows:

(a) For the first-order sensitivity of $D$ to $t, S(t)$ is equal to -1 . However, the higher order sensitivity $S h(t)$ changes approximately from -1 to -0.5 when time changes from $0 \%$ to $100 \%$.

(b) The increase and decrease in $D$ are asymmetrical with $t ; D$ changes much more with a decrease in $t$ than an increase in $t$ that are relatively equal in value. For example, a $50 \%$ reduction in $\mathrm{t}$ increases $D$ by $100 \%$, while a $50 \%$ increase in $\mathrm{t}$ reduces $D$ by about $33 \%$.

Therefore, $t$ should be assessed precisely in practice, since variations in $t$ of $50 \%$ could lead to variations in $D$ ranging from $-33 \%$ to $+100 \%$. This also implies that a small decrease in inspecting time greatly extends the value of the chloride diffusion coefficient. On the other hand, experiments for finding the chloride diffusion coefficient at a non-steady state should be observed as long as possible, e.g., up to one year.

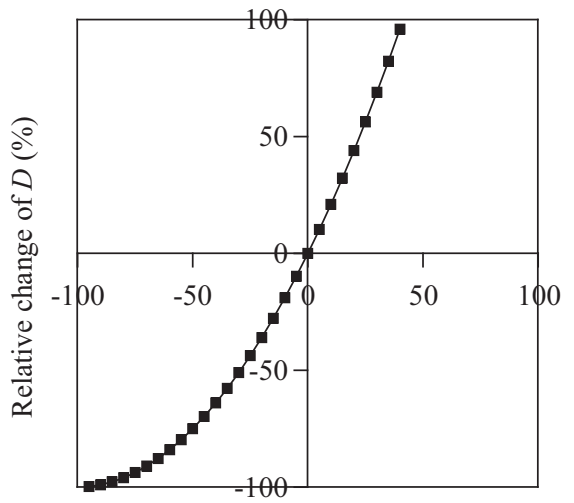

Relative variation of $d_{c}(\%)$

Fig. 3. Higher order impact of $\Delta D / D$ against $\Delta d_{c} / d_{c}$.

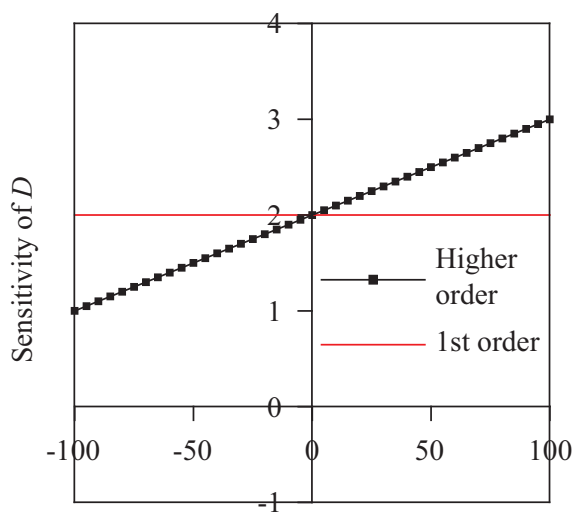

Relative variation of $d_{c}(\%)$

Fig. 4. Higher order and $1^{\text {st }}$ order sensitivity of chloride diffusion coefficient $D$ to inspecting depth $d_{c}$.

\section{Sensitivity of Chloride Diffusion Coefficient $D$ to Inspecting Concrete Depth $\boldsymbol{d}_{\boldsymbol{c}}$}

Eqs. (21) and (22) show the correlation between $\Delta D / D$ and $\Delta d_{c} / d_{c}$, the higher order impact of $\Delta D / D$ and $\Delta d_{c} / d_{c}$ is plotted in Fig. 3, and the sensitivity $S\left(d_{c}\right)$ of $D$ to $d_{c}$ is plotted in Fig. 4; these resulted in the following observations:

(a) For the first-order sensitivity of $D$ to $d_{c}, S\left(d_{c}\right)$ is equal to -2 . However, the higher order sensitivity $\operatorname{Sh}(t)$ changes approximately from 1 to 3 when $d_{c}$ changes from $-100 \%$ to $100 \%$.

(b) The increase and decrease in $D$ are asymmetrical with $d_{c}$, i.e. an increase in $d_{c}$ induces a much greater increase in $D$ than a decrease in $d_{c}$ at the same percentage, e.g., a 50\% increase in $d_{c}$ increases $D$ by $125 \%$, while a decrease in $d_{c}$ by $50 \%$ reduces $D$ by $75 \%$.

(c) Compared to $t, d_{c}$ has a larger impact on $D$, e.g., the variations in $d_{c}$ for $\pm 50 \%$, (which is a small variation in practice) could lead to variations in $D$ from $-75 \%$ to $+125 \%$. Therefore, $d_{c}$ should be precisely assessed in practice. 


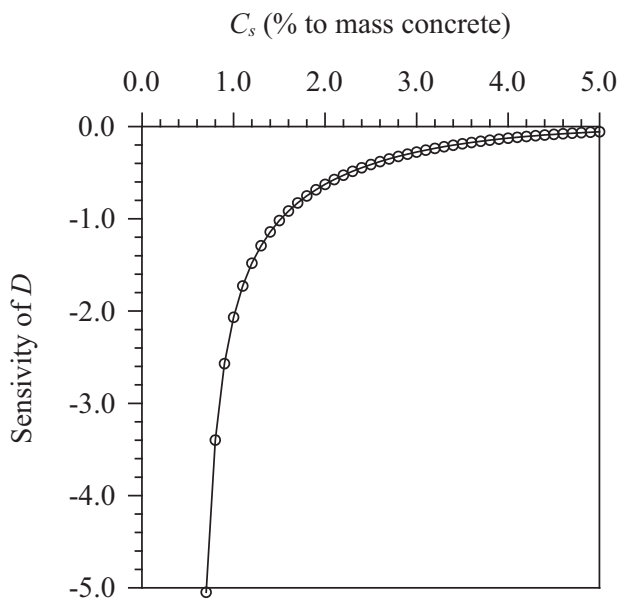

Fig. 5. First-order sensitivity of chloride diffusion coefficient $D$ to chloride surface concentration $C_{s}$.

This also implies that a small increase in inspecting depth would greatly extend the value of the chloride diffusion coefficient.

\section{Sensitivity of Chloride Diffusion Coefficient $D$ to Surface Chloride Concentration $C_{s}$}

If the inspecting result of diffusion coefficient is assumed to be $D(C)=10^{-6} \times e^{-C}, C_{i}$ and $C_{r}$ are $0.01 \%$ and $0.5 \%$, respectively. Eq. (17) evaluates the effect of a relative change in $C_{s}$, and the correlation between the sensitivity of the chloride diffusion coefficient $S\left(C_{s}\right)$ and $C_{s}$ is plotted in Fig. 5. Thus, the following observations can be made:

(a) The value of $S\left(C_{s}\right)$ increases negative-exponentially as $C_{s}$ increases from $0.5 \%$, and the values of $S\left(C_{s}\right)$ start from less than -10 and increase asymptotically towards zero. This means that an increase in $C_{s}$ induces a much greater decrease in $\left|S\left(C_{s}\right)\right|$ than a decrease in $C_{s}$.

(b) The surface chloride concentration $C_{s}$ is lesser at lighter exposure conditions than at high exposure conditions. As shown in Fig. 5, the variations in $D$ are more significant for lighter exposure conditions, e.g., when $C_{s}$ is $0.6 \%$, $\left|S\left(C_{s}\right)\right|$ is -10 , and when $C_{s}$ is $1.5 \%,\left|S\left(C_{s}\right)\right|$ is -1.02 .

(c) The sensitivity of the chloride diffusion coefficient to the surface chloride concentration comes into effect when $C_{s}$ is greater than the concentration at the inspecting point $C_{r}$.

\section{Sensitivity of the Chloride Diffusion Coefficient $D$ to the Chloride Concentration at Inspecting Point $\boldsymbol{C}_{\boldsymbol{r}}$}

Assuming that the inspection result shows the diffusion coefficient as $D(C)=10^{-6} \times e^{-C}, C_{i}$ and $C_{s}$ are $0.01 \%$ and $5.0 \%$, respectively. Eq. (18) evaluates the effect of a relative change in $C_{r}$, and the correlation between the sensitivity of the chloride diffusion coefficient $S\left(C_{r}\right)$ and $C_{r}$ is plotted in Fig. 6; thus, the following observations can be made:

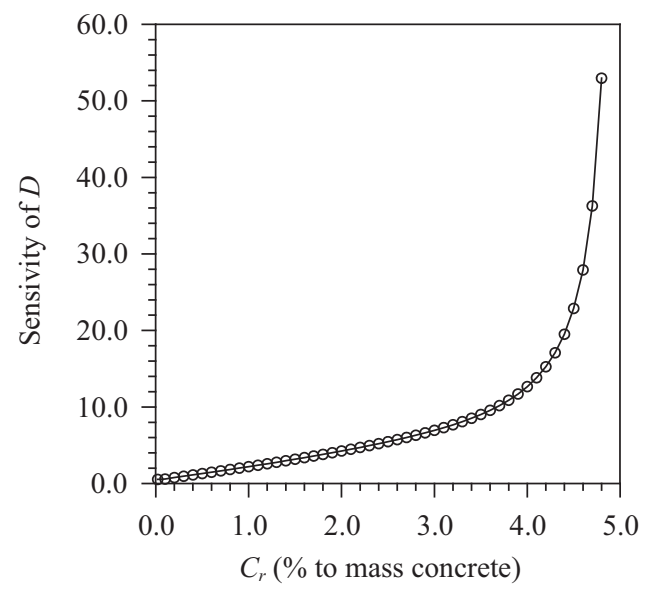

Fig. 6. First order sensitivity of chloride diffusion coefficient $D$ to chloride surface concentration at inspecting point $C_{r}$.

(a) The increase in $C_{r}$ induces a greater increase in $\left|S\left(C_{r}\right)\right|$ than an increase in $C_{r}$, e.g., when $C_{r}$ is $0.1 \%,\left|S\left(C_{r}\right)\right|$ is 0.58 , and when $C_{r}$ is $1.0 \%,\left|S\left(C_{r}\right)\right|$ is 2.2 and the ratio is $379 \%$. That means the increase rate of $S\left(C_{r}\right)$ is higher when $C_{r}$ is increased rather than decreased.

(b) The chloride concentration $C_{r}$ is less at lighter exposure conditions than at high exposure condition. As shown in Fig. 6, the variations in $D$ are more significant for high exposure conditions, and the increase in $C_{r}$ induces a greater increase in the value of the calculated result for $D$.

\section{CONCLUSIONS}

The sensitivity of the chloride diffusion coefficient to the four governing parameters of the time/depth dependent diffusion model - i.e., the inspecting time, inspecting depth, surface chloride concentration, and chloride concentration at the inspecting point - is investigated. These results have important applications in both modeling and practice.

1. The first-order sensitivities of the chloride diffusion coefficient to the inspecting time and depth are -1 and 2, respectively; they are independent of the surface chloride content and chloride at the inspecting point. The concrete depth has a higher impact on the diffusion coefficient than the inspecting time.

2. The duration of the non-steady state diffusion experiment should be long enough to get a practical result; otherwise, the value of the chloride diffusion coefficient will be overestimated. An overestimated chloride diffusion coefficient underestimates the durability of a concrete structure and may influence the accuracy of the engineering life-cycle cost estimation.

3. An increase in $d_{c}$ induces a much greater increase in $D$ than a decrease of $d_{c}$ for the same percentage, e.g., a $50 \%$ in- 
crease in $d_{c}$ increases $D$ by $125 \%$. This means that a small increase in inspecting depth greatly extends the value of the chloride diffusion coefficient. This also implies that if the inspecting point is at a shallow depth, the calculated result of the diffusion coefficient becomes smaller than if a deeper inspecting point is chosen.

4. An increase in $C_{s}$ induces a much greater decrease in $\left|S\left(C_{s}\right)\right|$, and a higher $C_{s}$ becomes closer to the saturated concentration induce by the outer environment. This implies that the impact of the inspecting result decreases to the minimum for the surface chloride concentration balance with the outside chloride concentration when concrete structures are immerged in or are in contact with a chloride environment, e.g. when $C_{s}$ is $1.5 \%,\left|S\left(C_{s}\right)\right|$ is -1.02 , and when $C_{s}$ is $3 \%,\left|S\left(C_{s}\right)\right|$ is -0.28 .

5. The increase $C_{r}$ induces a greater increase the value of the calculated result for $D$. It is critical to choose a reference chloride concentration to properly calculate diffusion coefficient predictions, e.g., when $C_{r}$ increases by $10 \%, D$ increases by $379 \%$.

\section{REFERENCES}

1. Alonso, C., Andrade, C., Castello, M., and Castro, P., "Chloride threshold values to depassivate reinforcing bars embedded in a standardized OPC mortar," Cement and Concrete Research, Vol. 30, pp. 1047-1055 (2000).

2. Boddy, A., Bentz, E., Thomas, M. D. A., and Hooton, R. D., "An over- view and sensitivity study of a multimechanistic chloride transport model," Cement and Concrete Research, Vol. 29, pp. 827-837 (1999).

3. Hohenbichler, M. and Rackwitz, R., "Sensitivity and importance measures in structural reliability," Civil Engineering Systems, Vol. 3, No. 4, pp. 203-209 (1986)

4. Liang, M. T., Huang, R., Feng, S. A., and Yeh, C. J., "Service life prediction of pier for the existing reinforced concete bridges in chlorideladen environment," Journal of Marine Science and Technology, Vol. 17, No. 4, pp. 312-319 (2009).

5. Oh, B. H. and Yang, I. H., "Sensitivity analysis of time-dependent behavior in PSC box girder bridges," Journal of structural Engineering, Vol. 126, pp. 171-179 (2000).

6. Sun, Y. M., Chang, T. P., and Liang, M. T., "Kirchhoff transformation analysis for determining time/depth dependent chloride diffusion coefficient in concrete," Journal of Material Science, Vol. 43, pp. 1429-1437 (2008).

7. Tzeng, C. Y. and Wu, C. Y., "On the design and analysis of ship stabilizimg fin controller," Journal of Marine Science and Technology, Vol. 8, No. 2, pp. 117-124 (2000).

8. Tzeng, C. Y., Wu, C. Y., and Chu, Y. L., "A sensitivity function approach to the design of rudder roll stabilization controller," Journal of Marine Science and Technology, Vol. 9, No. 2, pp. 100-112 (2001).

9. Williamson, G. S., Weyers, R. E., Brown, M. C., Ramniceanu, A., and Sprinkel, M. M., "Validation of probability-based chloride-induced corrosion service-life model," ACI Materials Journal, Title No. 105-M43, July-Aug., pp. 375-380 (2008).

10. Yeih, W. D., Huang, R., and Chang, J. J., "A study of chloride diffusion properties of concrete at early age," Journal of Marine Science and Technology, Vol. 2, pp. 61-67 (1994).

11. Zhang, J. and Lounis, Z., "Sensitivity analysis of simplified diffusionbased corrosion initiation model of concrete structures exposed to chlorides," Cement and Concrete Research, Vol. 36, pp. 1312-1323 (2006). 